\title{
LOS ARGUMENTOS LINGÜÍSTICOS DE KUHN
}

\author{
Julio C. ARMERO
}

\author{
Por ouestra cruda mano \\ aquella triste traducción furiosa \\ no tiene hueso sano \\ y vive sospechosa
}

(Hernando de Acuña)

Cuando por primera vez se publicó La Estructura de las Revoluciones Científicas, ésta violentaba la manera de hacer la filosofía de la ciencia. La modificación de la disciplina, hasta el punto de encontrar cómoda cabida en ella, fue uno de los efectos de la obra. Kuhn era principalmente un historiador que habia ideado una teoría del cambio científico y había introducido unos cuantos conceptos afortunados para describirlo.

La filosofía de la ciencia anterior a Kuhn, en la tradición establecida por el positivismo lógico y por Popper era en lo principal lógica aplicada, e incluso la base empírica era discutida atendiendo a las relaciones lógicas que se podrian establecer entre ella, según fuera caracterizada de una u otra manera, y la teoría. La lógica, incluida la lógica inductiva, sistematiza aspectos esenciales de la racionalidad $y$, puesto que la ciencia es una empresa racional, su naturaleza debería poder ser puesta en claro mediante el análisis lógico.

La débil relación con la historia de la ciencia era, como decía Feigl, que ésta habría de vindicar las reglas metodológicas que

Éndoxa: Series Filosóficas, no 9, 1997, UNED, Madrid:

Julio César Armero: Los argumentos lingüísticos de Kuhn.

pp. 125-137. 
propusiera la filosofía de la ciencia. Y, más en general, a ningún metodólogo le agradaría ver que las grandes realizaciones científicas, del pasado o del futuro, contravienen drásticamente sus prescripciones.

No voy a entrar en las razones obvias, y otras no tan obvias, por las que los filósofos de la ciencia buscaban vindicarse en la historia. Pero fue por esta vía por la que la obra de Kuhn se convirtió en un desafío para los filósofos de la ciencia. La Estructura de las Revoluciones Científicas declaraba que en la historia de la ciencia hay patrones de cambio científico que no se someten a las reglas de la tradición metodológica establecida por filósofos y otros teóricos.

El desacuerdo más sensible se produjo a propósito de la contrastación de teorías, y en especial, cuando sirve para decidir qué teoría es la mejor cuando hay dos en competencia.

La mayor parte de los estudios sobre la contrastación, tanto de Popper como de los positivistas lógicos, se ocupan del caso de una teoría que es confrontada con los enunciados, no problemáticos, de la base empírica.

De acuerdo con Kuhn, en cambio, esta clase de situaciones sólo ocurre durante los períodos de ciencia normal. Pero hay otra clase más interesante en la que la contrastación forma parte de la competencia entre dos teorías rivales. ${ }^{1}$

Para la concepción heredada (como Putnam la llama) es fácil concebir la confrontación entre varias teorías, y su resolución mediante experimentos cruciales. Cuando hay varias teorías en competencia, y unas no se reducen a otras, entonces deben tener consecuencias empíricas incompatibles. El procedimiento para decidir la rivalidad es extraer esas consecuencias y diseñar experimentos que adjudiquen la controversia. Esto se puede hacer porque los enunciados de la base empírica no son problemáticos;

1 T. Kuhn, "The Structure of Scientific Revolutions", en O. Neurath. R. Carnap y Ch. Morris, eds., Foundations of the Unity of Science, vol. II, Chicago, 1970, p. 207. Citado en adelante como SSR. 
esto es, se pueden establecer con independencia de las teorías sometidas a contrastación y son inteligibles y aceptables para los proponentes de teorías rivales.

Kuhn, por el contrario, sólo se interesa por el caso en que dos teorías son confrontadas, y no un número cualquiera de ellas, porque estos episodios tienen lugar en los períodos de transición de un paradigma a otro.

Pero la diferencia esencial de Kuhn con los anteriores filósofos de la ciencia consiste en su afirmación de que "los proponentes de paradigmas rivales no pueden entrar en un completo contacto con los puntos de vista de los demás" y que "la comunicación a través de la línea de división revolucionaria es necesariamente parcial". ${ }^{3}$ Esta dificultad de la comunicación ocurre porque los científicos adscritos a paradigmas rivales aplican la terminología a la naturaleza de manera diferente, y "por lo tanto, la superioridad de una teoría sobre otra no se puede probar en el debate". ${ }^{4}$ Esto quiere decir, como mínimo, que no hay enunciados básicos comunes que sean entendidos de la misma forma y aceptados por las tradiciones científicas separadas por una revolución.

En general, la fractura de la comunicación se produce porque los paradigmas son inconmensurables. Con este término Kuhn pretende aludir a un conjunto de hechos que cree haber observado en las transiciones revolucionarias:

1. Los problemas que cada tradición juzga interesante resolver pueden diferir. El nuevo paradigma tiene que intentar resolver los problemas que condujeron a la crisis del anterior, y también se ocupa de nuevos rompecabezas. Pero es frecuente que, además, algunos que resolvía el viejo paradigma no sean resueltos en el nuevo y sean juzgados ajenos a la disciplina.

2. La reinterpretación de instrumentos y de términos. Los términos y los experimentos entran, en el nuevo paradigma, en

\footnotetext{
2 SSR, p. 210.

${ }^{3}$ SSR, p. 211.

${ }^{4}$ SSR, p. 260.
} 
relaciones distintas de las que tenían en el viejo, de modo que los términos se conectan entre sí y con la naturaleza de un modo diferente.

3. Los científicos separados por una revolución viven en mundos diferentes. Singular tesis que tiene un valor expresivo o metafórico, a la vista de que el propio Kuhn asegura sensatamente en otro lugar que los científicos viven en el mismo mundo. ${ }^{5}$ Otra forma de esta tesis es que los científicos separados por crisis revolucionarias ven, en algunos casos importantes, cosas diferentes cuando se les presentan los mismos estímulos.

Tiene razón Kuhn, contra sus críticos, en que la inconmensurabilidad no implica la incomparabilidad de las teorías. Se necesita además suponer alguna otra premisa, por ejemplo, que un componente esencial de la comparación es diseñar y resolver experimentos cruciales. Pero no discutiremos esta cuestión.

De los tres aspectos de la inconmensurabilidad, el segundo es de naturaleza lingüística, y el tercero tiene efectos lingüísticos, porque "dos hombres que perciben la misma situación de forma diferente pero, sin embargo, usen el mismo vocabulario en su discusión, deben usar las palabras de forma diferente". 6

A su vez, las diferencias en la percepción dependen de las diferencias en los sistemas conceptuales. Esto es, la percepción conlleva un procesamiento descendente, como dicen los psicólogos, de los estímulos.

Las diferencias entre los sistemas conceptuales anterior y posterior a una revolución son descritas por Kuhn como el reagrupamiento de los objetos de que se ocupa la comunidad científica en conjuntos diferentes. Pero, "puesto que la mayoría de los objetos pertenecientes incluso a estos conjuntos alterados continúan agrupados juntos, generalmente se preservan los nombres de los conjuntos".

\footnotetext{
5 SSR, P. 191.

${ }^{6}$ SSR, p. 262.

7 SSR, p. 262.
} 
Si se preservan los nombres de los conjuntos pero éstos son diferentes, lo que tenemos son diferentes vocabularios. Kuhn asegura que los usuarios de estos vocabularios diferentes "responden al mismo estímulo con descripciones y generalizaciones diferentes". ${ }^{8}$

Pero está claro que sólo son incompatibles en apariencia. El argumento de Kuhn sólo muestra que se deben producir malentendidos muy semejantes a los que ocasionaría un traductor que tradujera del francés "doux" por "dulce" en todo contexto. Kuhn, en vez de darse cuenta de que ha producido un argumento débil, declara en una obra posterior que los problemas de traducir "doux" por "dulce" y "esprit" por "espíritu" "no son casos de ambigüedad, sino de disparidad conceptual entre el francés y el castellano". 9

En primer lugar, es bien dudoso que esta diferencia conceptual sea de las que afectan a la percepción y a las creencias. "Doux" o "douce" se aplican en francés a la miel, a los tejidos, a las comidas sosas, a los encantos de las personas, a las cuestas suaves y a los vientos ligeros. Pocos franceses creerán que las abejas podrian libar en un guante de satén o que les vaya a subir la glucosa por ascender una cuestecilla en bicicleta.

En segundo lugar, es dudoso que los franceses, ante estímulos douces produzcan generalizaciones que un castellano hablante no admitiera, salvo en el contexto de la poesía o los chistes. También es dudoso que hubiera un atomista francés lo bastante ingenuo como para buscar la clase de átomos (¿quizá redondos?) que hace que las cosas douces lo sean.

Las dificultades de comunicación se producen, según Kuhn, principalmente cuando están implicadas las partes del vocabulario de las teorías rivales que intervienen en una eventual contrastación

${ }^{8}$ SSR, p. 263.

9 T. Kuhn, "Conmensurabilidad, Comparabilidad y Comunicabilidad", en T. Kuhn, ¿Qué Son las Reooluciones Cientificas? y Otros Ensayos, trad. de J. Romo, Barcelona, 1989, p. 123. En adelante CCC. 
que decidiera entre ellas. La inconmensurabilidad entre las teorias no es siempre global, porque pueden quedar amplios segmentos de vocabulario relativamente poco afectados por los cambios. Pero precisamente las diferencias están presentes allí donde hay conflicto. ${ }^{10}$

Para salvar los problemas de comunicación, lo que los científicos "pueden hacer es reconocer que unos y otros son miembros de comunidades lingüísticas diferentes $\mathrm{y}$ entonces convertirse en traductores". ${ }^{11}$ Con ayuda del vocabulario compartido, los científicos pueden encontrar una vía para "elucidar sus problemas". ${ }^{12}$ "Esto es, cada uno puede intentar descubrir lo que el otro vería cuando se le presenta un estímulo al que su propia respuesta verbal sería diferente". ${ }^{13}$

Por el contexto en el que Kuhn habla, parece pensar que esto último es lo mismo que convertirse en un traductor. Pero, desde luego, que se sepa, el oficio de traductor alcanza como mucho a descubrir lo que el otro diría, no lo que veria. Y ése es el oficio del traductor inverso, del que traduce de su lengua nativa a la ajena.

En la misma página Kuhn cita a Quine como una autoridad en el tema de la traducción, pero le objeta que no pueda dar cuenta de "en qué medida el traductor debe ser capaz de describir el mundo al que se aplica el lenguaje sometido a traducción". ${ }^{14}$ Semejante crítica a Quine supone que la traducción de términos no está indeterminada y que la referencia no es inescrutable, o al menos no tanto como cree Quine. Éstas son conocidas tesis de Quine y Kuhn debería haber advertido, por lo menos, que él no. cree que la referencia esté indeterminada (o no tanto como dice Quine). Sin embargo, Kuhn dice que "Quine parece suponer que dos hombres que reciben el mismo estímulo tienen la misma

\footnotetext{
SSR, p. 263.

11 SSR, p. 264.

12 SSR, p. 264.

13 SSR, p. 264.

14 SSR, p. 264, nota 17.
} 
sensación" y que es por eso por lo que Quine no puede explicar las obligaciones que Kuhn asigna al traductor. ${ }^{15}$

Desde luego, lo que Quine parece suponer es que la sensación, en el sentido de Kuhn, ni es la misma ni deja de serlo, porque es inescrutable. Veamos, pues, cuál es el sentido que da Kuhn a sensación. En la página 182 de SSR Kuhn defiende la legitimidad de lo que él considera el uso corriente de "ver". Según este uso, las diferencias entre un aristotélico y Galileo cuando miran un péndulo son diferencias de visión o diferencias, dramáticamente expresado, entre sus mundos. Si es éste el sentido en el que ver es una sensación (cosa que Kuhn no aclara), pero que es en cualquier caso el sentido privilegiado del que dependen los argumentos de Kuhn que involucran la percepción, entonces la sensación es concebida por Kuhn como intencional y referencial, y entonces, de acuerdo con Quine, está indeterminada, tanto como la referencia o la traducción de términos.

Naturalmente no se trata en este artículo de restituir una adecuada interpretación de Quine frente a las confusiones de Kuhn, sino de detectar éstas. Que la lectura que hace Kuhn de Quine es vacilante se advierte en "Reflections on my Critics", 16 donde Kuhn parece haberse arrepentido de imputar a Quine la determinación de las sensaciones. Dice que los historiadores y lingüistas, cuando se ocupan de la traducción, se interesan por "cómo los hablantes nativos ven el mundo, qué clase de categorias ontológicas despliegan". 17

Porque "parte de aprender un lenguaje o una teoría es aprender a describir el mundo con el que funciona el lenguaje o la teoría". ${ }^{18}$ De modo que Kuhn sigue atribuyendo al traductor las mismas obligaciones que en SSR, pero, generosamente, descarga a Quine

15 SSR, p. 264, nota 17.

16 T. Kuhn, "Reflections on my Critics", en I. Lakatos y A. Musgrave, Criticism and the Growth of Knowledge, Cambridge, 1976. En adelante Reflections.

17 Reflections, p. 269.

18 Reflections, p. 270. 
de culpas. Quine no se ocupa de este aspecto, pero dice Kuhn que tampoco tiene por qué ocuparse, por no se sabe qué curiosa limitación de los propósitos de Quine.

La traducción es, según Kuhn, un recurso que tienen los científicos para aproximarse a las teorías rivales, pero la traducción sólo es posible en un grado limitado. La traducción perfecta no existe. Kuhn cita Palabra y Objeto para autorizar la tesis de que son posibles traducciones de un mismo texto con diferentes valores de verdad, realizadas conforme a conjuntos de hipótesis analíticas compatibles con las mismas disposiciones de conducta verbal pero incompatibles entre sí. $Y$ el famoso ejemplo de "Gavagai" es ulteriormente elaborado en Reflections.

Así, procede a suponer el caso de que "en la comunidad examinada, los conejos cambian de color, longitud del pelo, andares, etc., durante la estación lluviosa". 19 Pasemos por alto el hecho de que la comunidad examinada no puede ser sino de conejos. Cuando aparece uno de estos animales, los hablantes dicen "Bavagai". "¿Hemos de traducir 'Bavagai' por 'conejo mojado', 'conejo peludo', 'conejo saltarín', todo a la vez?, ¿o debe el lingüista concluir que la comunidad nativa no ha reconocido que 'Bavagai' y 'Gavagai' se refieren al mismo animal?"20

El desaliño de la argumentación de Kuhn es patente. En su lectura de Palabra y Objeto no se ha enterado: a) de la convención que usan Quine y casi todos los filósofos del lenguaje de escribir con minúscula los nombres y con mayúscula las oraciones, b) de que "Gavagai" no es el nombre de un término, sino de una oración.

Mientras que la traducción de términos está siempre indeterminada, según la teoría de la traducción de Quine, la de oraciones observacionales no lo está. Puesto que las oraciones observacionales son decisivas, según una amplia tradición, para contrastar las teorías, Kuhn debería haber explicado si cree o no en la indeterminación de la traducción de oraciones observacionales. En cambio,

\footnotetext{
${ }^{19}$ Reflections, p. 286.

20 Reflections, p. 268.
} 
parece creer que la traducción de términos no está excesivamente indeterminada, porque encomienda a historiadores y lingüistas la tarea no sólo de traducirlos sino de "escrutar" su referencia.

Kuhn afirma acertadamente que el recurso a la traducción con que pueden contar los científicos, y del que hacen uso abundante los historiadores "no tiene por qué ser una versión completa en un lenguaje neutral ni siquiera de las consecuencias de las teorías". ${ }^{21}$ Fundamentalmente esto ocurre porque no hay un lenguaje neutral, como por ejemplo un lenguaje de datos sensoriales, porque todo lenguaje, incluso éste, conlleva alguna clase de teoría. Esto, como podemos observar, es una consideración aparte de la de que el mundo es segmentado de maneras distintas por los vocabularios de las tradiciones científicas rivales. No se trata sólo de agrupar los mismos objetos en diferentes conjuntos.

Kuhn desarrolla esta consideración en dos aspectos que complican la traducción. El primero se refiere a la simultaneidad e inseparabilidad del aprendizaje sobre la naturaleza y del lenguaje. Kuhn señala que ordinariamente el vocabulario se adquiere junto con "una batería mínima de generalizaciones que muestran cómo se aplica a la naturaleza, 22 aunque, desde luego, llegamos a aprender muchas más cosas que las necesarias para simplemente adquirir el vocabulario. A esto se añade que una gran parte del proceso de adquisición del conocimiento y del lenguaje tiene lugar por un medio no lingüístico, como es la ostensión. ${ }^{23}$

El segundo aspecto se refiere al caso de que los términos de género en una lengua sean atribuidos sobre la base de "reconocer características distinguibles que son desconocidas"24 para los hablantes de otra lengua y para las que esta lengua carece de terminología descriptiva.

\footnotetext{
21 Reflections, p. 268.

22 Reflections, p. 270.

23 Reflections, p. 271.

24 CCC, p. 107.
} 
Kuhn da mucha importancia al aprendizaje por ostensión de vocabulario a la vez que de hechos. Al fin y al cabo, cuando alguien aprende un paradigma aprende a la vez la resolución de un problema y el vocabulario en que el problema y la solución se formulan.

La principal dificultad de la traducción radical de oraciones, de acuerdo con Quine, procede de que en muchos casos no se puede discernir cuándo el hablante nativo asiente a una oración porque posee ciertos conocimientos sobre la situación o porque la oración es analítica.

La imposibilidad de distinguir entre analítico y sintético ha sido puesta de relieve por numerosos autores. Kuhn atribuye a Carnap la idea de que junto con el vocabulario se adquieren generalizaciones, aunque el mismo Campbell y luego Popper advirtieron sobre la conexión entre significados y generalizaciones. Sin embargo, la idea de que son indiscernibles ha sido elaborada fundamentalmente por Quine, y es una de sus posiciones más conocidas. En inteligencia artificial y traducción automática ha sido explotado esto por Bar-Hillel, entre otros. Pero el mismo Wittgenstein señala algo de este estilo cuando dice que para estar de acuerdo en los significados hay que estar de acuerdo en los hechos.

Hacia algo semejante a esto apunta Kuhn cuando dice que los científicos que usan los términos de manera diferente no están en desacuerdo simplemente en una materia de definición, o no de definición estipulativa. ${ }^{25}$

Tarski, en su teoría de la definición, propone que una definición contextual sea un tipo de enunciado bicondicional que se deriva de un conjunto de tesis aceptadas. Cuando hay desacuerdo acerca de una definición de este estilo, el desacuerdo no es simplemente un desacuerdo sobre estipulaciones. Parte de un desacuerdo en las tesis que se aceptan.

Con esto llegamos a un problema fundamental en Kuhn. Él no está dispuesto a afirmar que la diferencia en el caso de científicos

25 SSR, p. 193. 
que usan vocabularios distintos proceda de que están en desacuerdo en los hechos. Pero la única explicación de que usen el vocabulario de forma diferente y sea imposible la traducción es que estén en desacuerdo en los hechos. $Y$ si no se puede discernir si el desacuerdo es acerca de los hechos o de los significados, entonces está indeterminada.

Sugiere Kuhn, como mejor aproximación a la traducción, no tratar las afirmaciones anómalas del rival como meros productos del error. ${ }^{26}$ Ya se sabe que la única forma de empezar a traducir es aplicar el principio de caridad. Pero no podemos áplicarlo, o nos induce a error hacerlo, cuando positivamente estamos en desacuerdo acerca de los hechos con el hablante de la otra comunidad.

Por lo tanto, o bien reconocemos que las diferencias no son diferencias de definición estipulativa o bien aplicamos el principio de caridad. Mientras aplicamos el principio de caridad suponemos que el otro hablante comparte nuestras creencias. Pero eventualmente llegará un momento en que hayamos diseñado nuestras hipótesis analíticas y podamos atribuir al hablante errores en cuestiones de hecho. En la medida en que hagamos esto, habremos separado lo analítico de lo sintético. Un manual de hipótesis analíticas es un conjunto de decisiones acerca de cómo se traza la distinción en el lenguaje nativo. $Y$ es impensable construir un manual de hipótesis analíticas que tenga como consecuencia que no podamos atribuir ningún error en cuestiones de hecho al hablante nativo. Sería sobremanera complicado. Es posible que la historia de la filosofía y la historia de la ciencia sean actividades eternas porque los historiadores insisten en aplicar ilimitadamente el principio de caridad. Todo lo que no queramos atribuir al error lo tendremos que atribuir a cada vez más peculiares significados.

Pero si las proferencias anómalas las atribuimos a diferencias de definición no nominal, a diferencias en definiciones tarskianas, entonces las atribuimos a diferencias en cuestiones de hecho. Kuhn insiste en que las diferencias son sólo de definición (no acerca de

26 SSR, p. 264. 
los hechos), pero no de definición nominal. La única manera de compatibilizar ambas afirmaciones de Kuhn es advertir que la distinción analítico/sintético es borrosa. Pero el recurso a la traducción es el recurso a fijar de alguna manera la distinción analítico/sintético.

Un problema distinto es el que aparece si el hablante nativo conoce algo más que lo que conoce el traductor. Kuhn considera que los términos que son aprendidos por ostensión presentan a la traducción dificultades especiales. También aquéllos que son introducidos mediante una definición que aluda a rasgos desconocidos para el traductor. Los términos aprendidos por ostensión presentan un problema si la ostensión se hace sobre una situación que contiene algún rasgo ignorado por el traductor. En los dos casos los problemas de traducción proceden de que el nativo conoce algo que el traductor no conoce. La única solución para el traductor es incorporar ese nuevo conocimiento, si lo averigua, junto con, eventualmente, un nuevo vocabulario. En ocasiones deberá además renunciar a parte de su propio vocabulario. Es decir, la solución es modificar su lenguaje.

Kuhn, como todo el mundo, tiene algunas dificultades con la individuación de lenguajes. Distingue entre traducción e interpretación porque ha advertido (CCC) que cuando el nativo se equivoca, algunos de sus términos pueden ser considerados no referenciales. Si hacemos eso, en la traducción de un texto aparecerán blancos, y una traducción con huecos no es aceptable según criterios editoriales ordinarios ni según Kuhn.

Para llenar los espacios vacíos de la traducción debemos complicar intolerablemente nuestras hipótesis analíticas o modificar nuestro lenguaje y aclarar esas modificaciones en notas del traductor. Estas extensiones y supresiones deben ser consideradas virtuales. Mientras que en el caso de que el nativo posea conocimientos extraordinarios, las extensiones (y las supresiones) serán auténticas, esto es, con compromiso ontológico. En el lenguaje ordinario conviven términos que presuponen contradicciones con conocimientos aceptados. Además, el vocabulario del lenguaje 
ordinario crece, pero, en cierto sentido legítimo, no se desprende nunca de sus elementos. En esa medida, el lenguaje ordinario es relativamente difícil de individualizar, y no está claro si sus extensiones son auténticas o no, como no está claro que muchos de los términos que contiene sean tomados como referenciales o no.

- Las modificaciones del lenguaje son necesarias en lo que Kuhn llama "interpretaciones", que distingue de las traducciones o simples versiones del texto, sin aparato de notas. Me parece que una de las funciones de este aparato es introducir las modificaciones virtuales del lenguaje que serán luego utilizadas en la exposición o traducción. Pero el historiador de la ciencia, como el científico que intenta comprender el punto de vista de una tradición rival, debe distinguir si las modificaciones de su lenguaje son virtuales o sinceras. Pueden llevar a borrar la diferencia el uso de la cita indirecta y el principio de la caridad mal entendida, remedio excesivo contra la máxima de que la caridad empieza por uno mismo. 